\title{
High-Resolution Imaging of the Distributions of Cholesterol, Sphingolipids, and Specific Proteins in the Plasma Membrane with Secondary Ion Mass Spectrometry
}

\author{
Mary L. Kraft ${ }^{1}$, Peter K. Weber, ${ }^{2}$ Jessica F. Frisz ${ }^{1}$, Haley A. Klitzing, ${ }^{1}$ Robert Wilson, ${ }^{1}$ Ashley Yeager, ${ }^{1}$ \\ and Joshua Zimmerberg ${ }^{3}$ \\ 1. School of Chemical Sciences, University of Illinois, 600 S. Mathews Avenue, Urbana, IL 61801, \\ USA. \\ 2. Glenn T. Seaborg Institute, Lawrence Livermore National Laboratory, 7000 East Ave., Livermore CA \\ 94551, USA. \\ ${ }^{3}$ Eunice Kennedy Shriver National Institute of Child Health and Human Development, National \\ Institutes of Health, Bethesda, MD 20892, USA.
}

The plasma membrane is a selectively permeable lipid bilayer that separates cells from their surroundings. Numerous different lipid species, cholesterol, and a variety of different proteins form the plasma membranes of mammalian cells. One class of lipids, the sphingolipids, and their metabolites serve both as structural components in the plasma membranes of mammalian cells, and as bioactive signaling molecules that modulate fundamental cellular processes. Though segregation of the sphingolipids into distinct membrane domains is likely essential for cellular function, the sphingolipid distribution within the plasma membrane and the mechanisms that regulate it are poorly understood.

To overcome these disadvantages, we pioneered the use of high-resolution SIMS, performed with a Cameca NanoSIMS 50, for directly imaging metabolically incorporated, stable isotope-labeled lipids in actual cell membranes. The NanoSIMS 50 is a state-of-the-art magnetic sector secondary ion mass spectrometer that can image the elemental and isotopic composition at the surface (top $5 \mathrm{~nm}$ ) of a sample with high sensitivity and as high as 50-nm-lateral resolution. In order to visualize the cholesterol and sphingolipids in the plasma membrane, we used metabolic labeling with ${ }^{15} \mathrm{~N}$-sphingolipid precursors $\left({ }^{15} \mathrm{~N}\right.$-sphingosine and ${ }^{15} \mathrm{~N}$-sphinganine) and ${ }^{18} \mathrm{O}$-cholesterol to selectively incorporate distinct stable isotopes, ${ }^{15} \mathrm{~N}$ and ${ }^{18} \mathrm{O}$, into the sphingolipids and cholesterol, respectively, in living mouse fibroblast cells. Then we chemically fixed the cells with a method that does not alter membrane lipid distribution $[1,2]$. Well-preserved cells with normal morphologies are identified by imaging with low voltage secondary electron microscopy (SEM). To increase secondary ion yields, the cell samples are coated with a thin (3-nm) iridium layer that does not alter the lipid distribution on the cells. Then we used a Cameca NanoSIMS 50 instrument to map the lipid-specific isotope enrichments on their surfaces better than 100-nm-lateral resolution.

Using this approach, we have previously shown that the ${ }^{15} \mathrm{~N}$-sphingolipids are enriched within distinct domains in the plasma membranes of fibroblast cells [2]. Here we report how we have used this approach to probe the mechanisms responsible for this sphingolipid organization. We investigated whether the sphingolipid domains are dependent on cholesterol-sphingolipid interactions, or the diffusion barriers that are established by the cytoskeleton and its associated membrane proteins by using SIMS to image the effects of cholesterol depletion and actin depolymerisation on ${ }^{15} \mathrm{~N}$-sphingolipid distribution in the plasma membrane [2,3]. We also assessed whether these ${ }^{15} \mathrm{~N}$-sphingolipid domains are co-localized with hemagglutinin, a specific membrane protein that is thought to have an affinity for sphingolipid-enriched membrane domains. Our results indicate that the sphingolipid organizations in 
the plasma membranes of fibroblasts are dependent on the cytoskeleton, but not on favorable interactions with cholesterol or hemagglutinin.

\section{References}

[1] HA Klitzing., et al., Secondary Ion Mass Spectrometry Imaging of Biological Membranes at High Spatial Resolution. In Methods in Molecular Biology: Nanoimaging Methods and Protocols, Sousa, A. A., and Kruhlak, M. J., (eds.) Humana Press, Totowa, New Jersey, (2013), Vol. 950, pp 483.

[2] JF Frisz, et al., Proc. Nat. Acad. Sci. U.S.A. (2013) 110 (8), E613.

[3] JF Frisz, et al., J. Biol. Chem. (2013) 288 (23), 16855.

[4] The authors acknowledge funding from the Burroughs Wellcome Fund, the Intramural Program of the NICHD, NIH, the NIH Training Program in the Chemistry-Biology Interface T32 GM070421, the NSF under CHE-1058809, and Lab Directed Research and Development funding to LLNL. Dr. Kaiyan Lou is thanked for the synthesis of the ${ }^{15} \mathrm{~N}$-sphingolipid precursors and ${ }^{18} \mathrm{O}$-cholesterol. Work at LLNL was supported by Lab Directed Research and Development funding and performed under the auspices of the U.S. DOE. 